\title{
Facile preparation of self-assembled MXene@Au@CdS nanocomposite with enhanced photocatalytic hydrogen production activity
}

\author{
Juanjuan Yin ${ }^{1,2}$, Fangke Zhan ${ }^{2}$, Tifeng Jiao ${ }^{1,2 *}$, Wenhan Wang ${ }^{3}$, Guangcong Zhang ${ }^{2}$, Jinghua Jiao ${ }^{2}$,
} Guiyuan Jiang $^{3^{*}}$, Qingrui Zhang ${ }^{2}$, Jianmin $\mathrm{Gu}^{2}$ and Qiuming Peng ${ }^{1}$

\begin{abstract}
Photocatalytic hydrogen production is considered a promising approach to generating clean sustainable energy. However, the conventional co-catalyst (e.g., Pt) used in photocatalytic hydrogen production is high-cost and difficult to obtain. Here, we designed and prepared a ternary nanocomposite MXene@Au@CdS, which can be used in the field of efficient and excellent photocatalytic hydrogen production. The MXene@Au@CdS has a hydrogen production rate of $17,070.43 \mu \mathrm{mol} \mathrm{g}^{-1} \mathrm{~h}^{-1}$ (tested for $2 \mathrm{~h}$ ), which is 1.85 times that of pure CdS nanomaterials. The improved hydrogen production performance of the MXene@Au@CdS is attributed to: (i) MXene provides more active adsorption sites and reaction centers for Au and CdS nanoparticles; (ii) the synergistic effect of Au's strong surface plasmon resonance expands the optical response range of CdS. Therefore, this work solves the problem of the solid connection between the surface functional groups of photocatalyst, and achieves rapid interface charge transfer and long-term stability during the hydrogen production.
\end{abstract}

Keywords: nanocomposite, MXene, photocatalytic hydrogen production

\section{INTRODUCTION}

As technology advances, the environmental crisis and growing energy problems require clean and eco-friendly renewable energy. Photocatalytic hydrogen production is considered to be a promising approach to clean environments and sustainable energy, and has attracted widespread attention in the industry [1-3]. In addition, the use of solar energy to generate hydrogen from water is expected to be a strategy to solve global energy problems [4]. In particular, photocatalytic water decomposition using semiconductor photocatalysts has shown great potential. In recent years, cocatalysts have been promising in enhancing the activity and stability of photocatalysts [58]. However, the cocatalyst (e.g., Pt) used for hydrogen evolution generally is faced with high cost and difficulty in production, and the commercializing of the photocatalyst is a challenge. Therefore, it is crucial to find an inexpensive and highly active cocatalyst for the process of utilizing solar energy to produce hydrogen.

CdS has been extensively studied due to its visible light response and suitable energy band alignment for water decomposition $[9,10]$. However, pure CdS particles tend to aggregate and show high photo-generated charge recombination rate, resulting in shrinking surface area and inferior photocatalytic performance. Therefore, in order to address these issues, some studies have achieved efficient photocatalytic performance by adding various cocatalysts [11]. Among them, precious metal nanoparticles (such as Au, Ag) are effective co-catalysts to enhance the photocatalytic activity of CdS due to their local surface plasmon resonance [12]. The excited electrons on the Au nanoparticles could enter the conduction band of CdS by exciting local surface plasmon resonance, thereby improving the photocatalytic activity. In addition, the position of the noble metal also greatly affects the photocatalytic hydrogen production performance [13]. Metal/semiconductor heterostructures possess prominent

\footnotetext{
${ }^{1}$ State Key Laboratory of Metastable Materials Science and Technology, Yanshan University, Qinhuangdao 066004, China

${ }^{2}$ Hebei Key Laboratory of Applied Chemistry, School of Environmental and Chemical Engineering, Yanshan University, Qinhuangdao 066004, China

${ }^{3}$ State Key Laboratory of Heavy Oil Processing, China University of Petroleum Beijing, Beijing 102249, China

* Corresponding authors (emails: tfjiao@ysu.edu.cn (Jiao T); jianggy@cup.edu.cn (Jiang G))
} 
role in regulating the charge transfer in photocatalysis. Xiao's group [14,15] have done a series of studies on photooxidation catalysis. For example, Zeng et al. $[14,15]$ prepared a cascaded charge transfer channel in a multilayer photoanode by in-situ layer-by-layer construction to achieve plasma-induced photoelectrochemical water oxidation. Li et al. $[16,17]$ investigated the general self-assembly of metal/metal chalcogenide heterostructures induced by surface linkers regulating multipliable photoreduction catalysis with adjustable charge flow.

Naguib et al. [18] reported a two-dimensional (2D) transition metal carbide/carbonitride (MXene) and added a new member to the $2 \mathrm{D}$ material family. MXene possesses a large number of hydrophilic functional groups $(-\mathrm{OH}$ and $-\mathrm{O})$ on its surface which promote its strong interaction with water molecules and make it easy to establish strong connections with various semiconductors. The excellent metal conductivity of MXene ensures its effective carrier transfer, and the exposed metal sites (such as $\mathrm{Ti}, \mathrm{Nb}$, or $\mathrm{V}$ ) on MXene are more likely to undergo redox reactions than carbon materials [19]. In addition, MXene could stably function in aqueous solution. The above excellent properties render MXene a promising photocatalytic material. MXene materials demonstrate excellent physical and chemical properties such as abundant surface groups, strong electrical conductivity, excellent ductility and flexibility, which are applied in the fields of energy storage and conversion, air pollution and sewage treatment, electromagnetic shielding [20], etc. Yang et al. [21] reported that the velocity of holes on MXene were much higher than that of electrons according to the calculation results from density functional theory. Ran et al. [22] synthesized a $\mathrm{CdS} / \mathrm{Ti}_{3} \mathrm{C}_{2}$ composite and applied it to photolysis of hydrogen under visible light. The experimental results show that $\mathrm{Ti}_{3} \mathrm{C}_{2}$ is an excellent cocatalyst, which greatly enhances the photohydrolysis effect of CdS.

Here, we designed a new and efficient three-component $\mathrm{Ti}_{3} \mathrm{C}_{2}$ MXene@Au@CdS heterostructure for efficient photocatalytic hydrogen production under visible light. The Au nanoparticles have strong surface plasmon resonance, and hence they can be used as sensitizers to improve the photocatalytic efficiency and extend the optical response spectrum of $\mathrm{CdS}$. $\mathrm{Ti}_{3} \mathrm{C}_{2}$ MXene provides a stable matrix for the dispersion of the nanoparticles with high-efficiency charge separation. The synergistic effect from CdS to $\mathrm{Ti}_{3} \mathrm{C}_{2}$ MXene migration could accelerate the photocatalytic process. Moreover the $-\mathrm{O}$ terminating groups on $\mathrm{Ti}_{3} \mathrm{C}_{2}$ MXene can facilitate the release of $\mathrm{H}_{2}$. Therefore, this study purposed a strategy to obtain rich active surfaces for strong bonds with the photocatalyst, rapid transfer of interface charges and long-term stability. On the other hand, it provides MXene with a new application. Besides, this stable and cost-effective semiconductor photocatalyst could effectively realize solar water decomposition.

\section{EXPERIMENTAL SECTION}

\section{Materials}

Ethanol $\left(\mathrm{C}_{2} \mathrm{H}_{5} \mathrm{OH}\right)$ and chloroauric acid tetrahydrate $\left(\mathrm{HAuCl}_{4} \cdot 4 \mathrm{H}_{2} \mathrm{O}\right)$ were obtained from Sinopharm Holding Chemical Reagent Co., Ltd. (Beijing, China). Dimethyl sulfoxide (DMSO) and hydrofluoric acid were purchased from Tianjin Chemical Industry. Ultrapure water was prepared by using a Milli-Q Millipore filtration system (Millipore Co., Bedford, Massachusetts, USA). All chemicals were used as received without further purification.

\section{Preparation of MXene}

The MXene was prepared according to previous report [18]. Briefly, the MXene was obtained by etching away the $\mathrm{Al}$ layer of $\mathrm{Ti}_{3} \mathrm{AlC}_{2}$ at $40^{\circ} \mathrm{C}$ with $\mathrm{HF}$. After HF treatment, the sample was washed with demonized water until the $\mathrm{pH}$ value of the solution was around 6 , and then dried under vacuum at $80^{\circ} \mathrm{C}$ for $24 \mathrm{~h}$ to obtain $\mathrm{Ti}_{3} \mathrm{C}_{2}$ MXene.

\section{Synthesis of $\mathrm{Ti}_{3} \mathrm{C}_{2}$ MXene@Au composite}

The $\mathrm{Ti}_{3} \mathrm{C}_{2}$ MXene@Au composite was prepared by selfreduction method. Briefly, $100 \mathrm{mg}$ of $\mathrm{Ti}_{3} \mathrm{C}_{2}$ MXene was added to $100 \mathrm{~mL}$ of ultrapure water and stirred. Next, $3 \mathrm{~mL}$ of $\mathrm{HAuCl}_{4}\left(0.1 \mathrm{~mol} \mathrm{~L}^{-1}\right)$ was slowly added to the above suspension with constant stirring to trigger the selfreduction. After about half an hour, the mixture was centrifuged and washed several times with ultrapure water. Finally, lyophilization was carried out at $-60^{\circ} \mathrm{C}$ for $48 \mathrm{~h}$ to obtain the $\mathrm{Ti}_{3} \mathrm{C}_{2}$ MXene@Au composite.

\section{Preparation of MXene@Au@CdS composite}

First, $0.04 \mathrm{~g}$ of $\mathrm{Ti}_{3} \mathrm{C}_{2}$ MXene@Au solid powder was added to $35 \mathrm{~mL}$ of DMSO and stirred for about $20 \mathrm{~min}$, and then $0.1 \mathrm{~g}$ of cadmium acetate was put into the solution and continuously stirred for about $2 \mathrm{~h}$. Next, the mixed solution was transferred to a $50-\mathrm{mL}$ autoclave and maintained at $180^{\circ} \mathrm{C}$ for $12 \mathrm{~h}$. After cooling naturally, the mixed solution was taken out, and the supernatant was removed, leaving a precipitate, washed several times with ethanol, and collected by centrifugation. Then, $40 \mathrm{~mL}$ of ethanol was added to the precipitate, and the mixture was transferred to an autoclave, and maintained at $100^{\circ} \mathrm{C}$ for 
$20 \mathrm{~h}$. Finally, the sample was washed several times with ultrapure water to finally obtain $\mathrm{Ti}_{3} \mathrm{C}_{2}$ MXene@Au@CdS composite. $\mathrm{Ti}_{3} \mathrm{C}_{2}$ MXene@CdS was prepared in the same manner with $\mathrm{Ti}_{3} \mathrm{C}_{2}$ MXene as the reactant.

\section{Photocatalytic hydrogen production test}

The hydrogen production performance of the prepared samples were tested under visible light $(>420 \mathrm{~nm}$ ) from a 300W Xe arc lamp (Beijing Perfect Light Co., Ltd.) with a $420 \mathrm{~nm}$ cut filter. Generally, $4 \mathrm{mg}$ of the prepared photocatalyst was dispersed in $80 \mathrm{~mL}$ of a mixed aqueous solution of $0.35 \mathrm{~mol} \mathrm{~L}^{-1} \mathrm{Na}_{2} \mathrm{~S}$ and $0.25 \mathrm{~mol} \mathrm{~L}^{-1} \mathrm{Na}_{2} \mathrm{SO}_{3}$. In addition, a solution with $1 \mathrm{wt} \%$ of Pt promoter was added to the photocatalyst. Finally, the amount of $\mathrm{H}_{2}$ produced was measured by using a gas chromatograph (GC) equipped with a thermal conductivity detector (TCD).

\section{Photoelectric property test}

Photoelectrochemical measurements were performed by using a CHI660 electrochemical workstation (Solartron) with a standard three-electrode configuration, and the prepared composite nanomaterials were used as photoanodes with $\mathrm{Pt}$ foil and $\mathrm{Ag} / \mathrm{AgCl}$ electrodes as the counter and reference electrodes, respectively, and $\mathrm{Na}_{2} \mathrm{SO}_{4}$ $\left(1 \mathrm{~mol} \mathrm{~L}^{-1}\right)$ as the electrolyte. The simulated solar light source was a combination of a $300 \mathrm{~W}$ xenon lamp and an
AM 1.5G filter (Newport). Electrochemical impedance spectroscopy (EIS) was tested in a $1 \mathrm{~mol} \mathrm{~L}^{-1} \mathrm{Na}_{2} \mathrm{SO}_{4}$ solution with a direct current bias of $0.6 \mathrm{~V}$ against $\mathrm{Ag} / \mathrm{AgCl}$ in the frequency range of $10^{-1}$ to $10^{5} \mathrm{~Hz}$, where the tested alternative-current voltage was $10 \mathrm{mV}$.

\section{Characterization}

The CdS, $\mathrm{Ti}_{3} \mathrm{C}_{2}$ MXene and $\mathrm{Ti}_{3} \mathrm{C}_{2}$ MXene-based nanocomposite were characterized with scanning electron microscope (SEM), transmission electron microscope (TEM, HT7700, High-Technologies Corp., Japan) and high-resolution TEM (HRTEM, JEM-2100F, JEOL). Xray diffraction (XRD) was obtained on an X-ray diffract meter equipped with a $\mathrm{CuKa} \mathrm{X}$-ray radiation source and a Bragg diffraction device (SMART LAB, Rigaku, Japan). A NETZSCH STA 409 PC Luxxsi simultaneous thermal analyzer (Netzsch Instruments Manufacturing Co., Ltd., Germany) was used for thermo gravimetric (TG) analysis of the samples. UV-visible diffuse reflectance spectra (DRS) were obtained on a UV-visible spectrophotometer (Hitachi U3010) with $\mathrm{BaSO} 4$ as the substrate. X-ray photoelectron spectroscopy (XPS) data was obtained with Thermo Scientific ESCALab 250Xi under $200 \mathrm{~W}$ monochromatic AlKa radiations. The photocatalytic activity test was performed on a Labsolar II photocatalytic water analysis device produced by Beijing Perfila Co., Ltd.

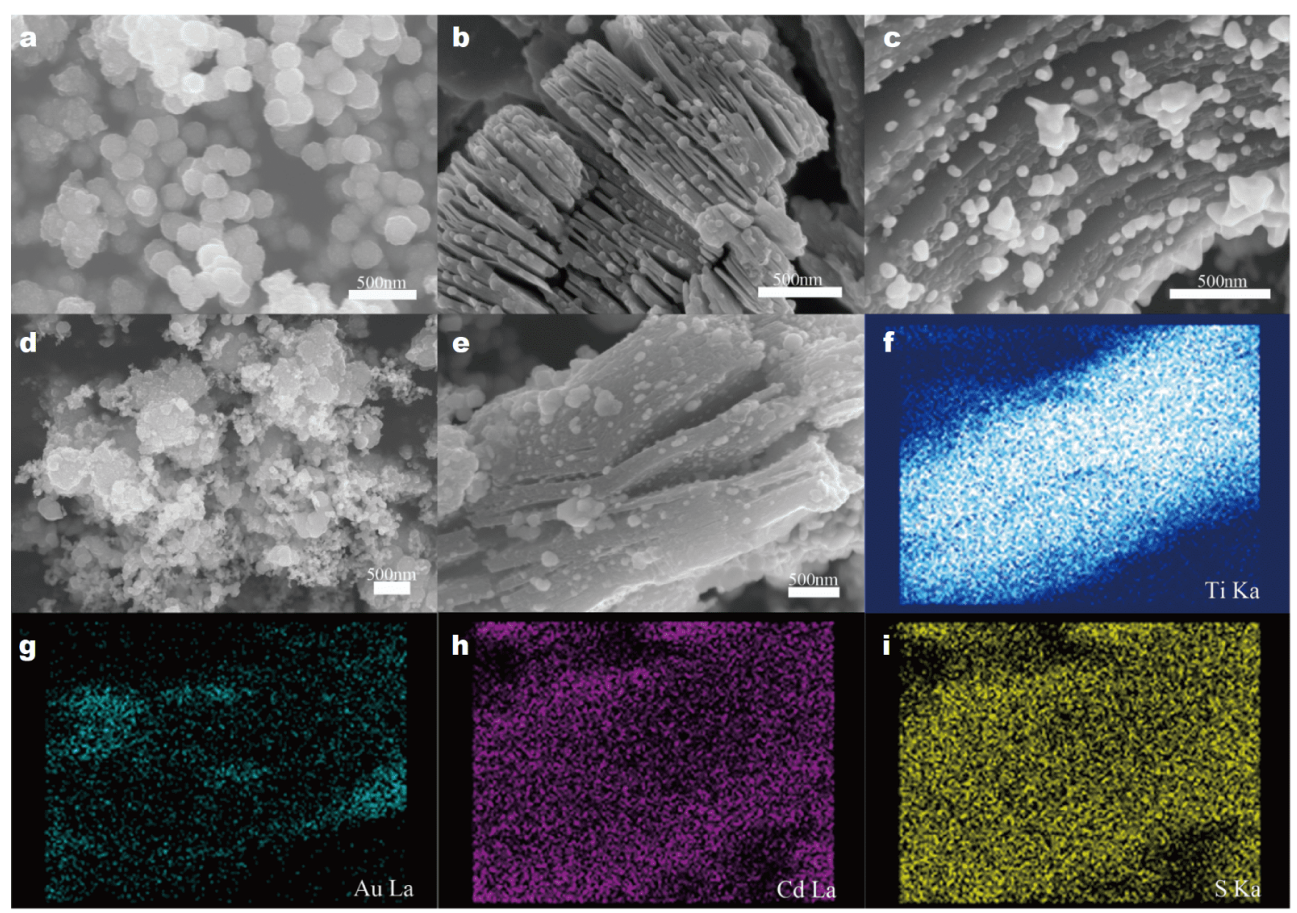

Figure 1 SEM images of (a) CdS, (b) MXene, (c) MXene@Au, (d) MXene@CdS and (e) MXene@Au@CdS; (f-i) the elemental mappings of MXene@Au@CdS. 


\section{RESULTS AND DISCUSSION}

\section{Structural characterization}

As shown in Fig. 1a, the size of CdS nanoparticles is around $150 \mathrm{~nm}$. Fig. $1 \mathrm{~b}$ shows the uniform layer structure of MXene, similar to the accordion [23]. When the chloroauric acid solution was added to a $\mathrm{Ti}_{3} \mathrm{C}_{2}$ MXene solution of a certain concentration, the Au nanoparticles were obtained by the self-reduction and uniformly dispersed on the $\mathrm{Ti}_{3} \mathrm{C}_{2}$ MXene, effectively preventing the aggregation of $\mathrm{Au}$ nanoparticles during the reduction process. The size of the $\mathrm{Au}$ nanoparticles is about 80-100 nm, as shown in Fig. 1c. CdS nanoparticles in-situ grew by adding cadmium acetate to the solution of $\mathrm{Ti}_{3} \mathrm{C}_{2}$ MXene@Au. As shown in Fig. 1d, the sheet of MXene is not very obvious and the surface becomes rough after the process of generating CdS. Fig. 1e shows that the prepared $\mathrm{Au}$ nanoparticles and CdS nanoparticles uniformly disperse on the MXene layer with no large-scale aggregation. The MXene not only provides sites for the growth of $\mathrm{Au}$ and $\mathrm{CdS}$ nanoparticles, but also reduces the aggregation of particles during the preparation process. As shown in Fig. 1f $-\mathrm{i}$, the mapping images of MXene $@ A u @ C d S$ show the presence of Ti, Au, Cd, and S, and most of $\mathrm{Au}$ and $\mathrm{CdS}$ nanoparticles are loaded on the surface of MXene or between the MXene layers.

As shown in Fig. 2a, the CdS nanoparticles exhibit a uniform spherical structure with an average diameter of about $150 \mathrm{~nm}$. MXene shows a typical layered structure with a uniform thickness (Fig. 2b). As shown in Fig. 2c, d, $\mathrm{Au}$ and $\mathrm{CdS}$ particles are reduced and uniformly attached to the surface of the MXene and each layer. Fig. 2e is a topographical view of the MXene@Au@CdS showing no significant aggregation, indicating that MXene is a good

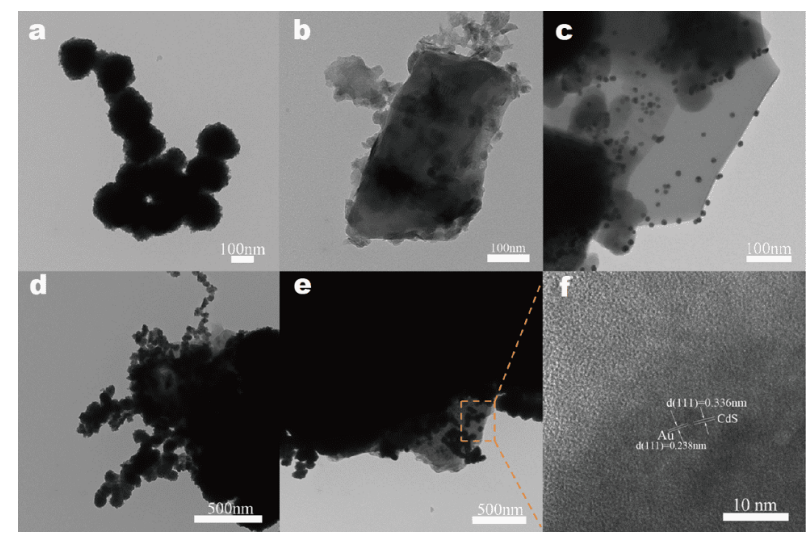

Figure 2 TEM images of (a) CdS, (b) MXene, (c) MXene@Au, (d) MXene@CdS, (e) MXene@Au@CdS and HRTEM image of (f) MXene@Au@CdS. substrate for dispersion growth. Fig. $2 \mathrm{f}$ is an HRTEM image of the heterostructure MXene@Au@CdS. The lattice spacing of the (111) crystal plane of CdS and the lattice spacing of the (111) crystal plane of $\mathrm{Au}$ in the HRTEM image are 0.336 and $0.238 \mathrm{~nm}$, respectively, which are consistent with the data corresponding to the peak positions of CdS and Au in XRD. These results indicate that tight connections are formed between MXene, $\mathrm{Au}$ and $\mathrm{CdS}$, which contribute to charge separation and transfer [24]. The energy dispersive X-ray spectroscopy (EDS) test of MXene@Au@CdS was performed, as shown in Fig. 3 [25,26], further confirming the heterostructure of MXene@Au@CdS and consistent with the TEM results.

XRD was used to characterize the prepared MXene, MXene@Au, MXene@CdS, CdS, and MXene@Au@CdS, as shown in Fig. 4a. For pure MXene, the diffraction peaks well match to the MXene reported [27]. In the curve of the MXene@Au@CdS, the peaks at $26.64^{\circ}, 44.44^{\circ}$ and $51.86^{\circ}$ can be attributed to the (111), (220) and (311) of CdS. Furthermore, the peaks at $38.20^{\circ}, 64.61^{\circ}$ and $77.80^{\circ}$ are assigned to the corresponding (111), (220) and (331) Au crystal faces. Therefore, it confirms that the nanocomposite MXene@Au@CdS has been successfully prepared [28].

The thermal stability of the obtained CdS, MXene@CdS and MXene@Au@CdS nanocomposites can be analyzed by TG under nitrogen [29-33], as shown in Fig. 4b. As the temperature gradually increases, the weight loss of the materials also gradually increases [34-36]. When the temperature rose to $400^{\circ} \mathrm{C}$, the weight loss of the material reached the largest, with about $4.5 \%, 4 \%$ and $1.5 \%$ loss for CdS, MXene@CdS and MXene@Au@CdS, respectively. For CdS, the sequential addition of MXene and Au en-

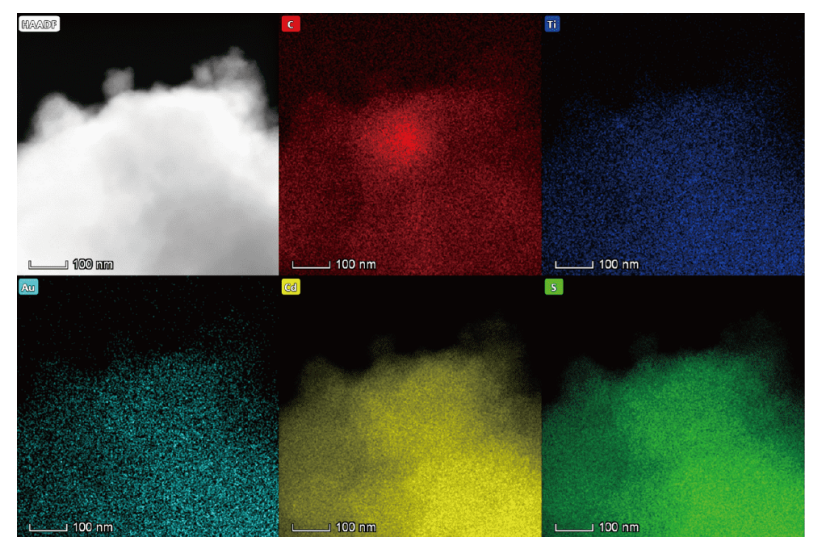

Figure 3 TEM images and elemental mapping images of MXene@Au@CdS nanocomposite. 

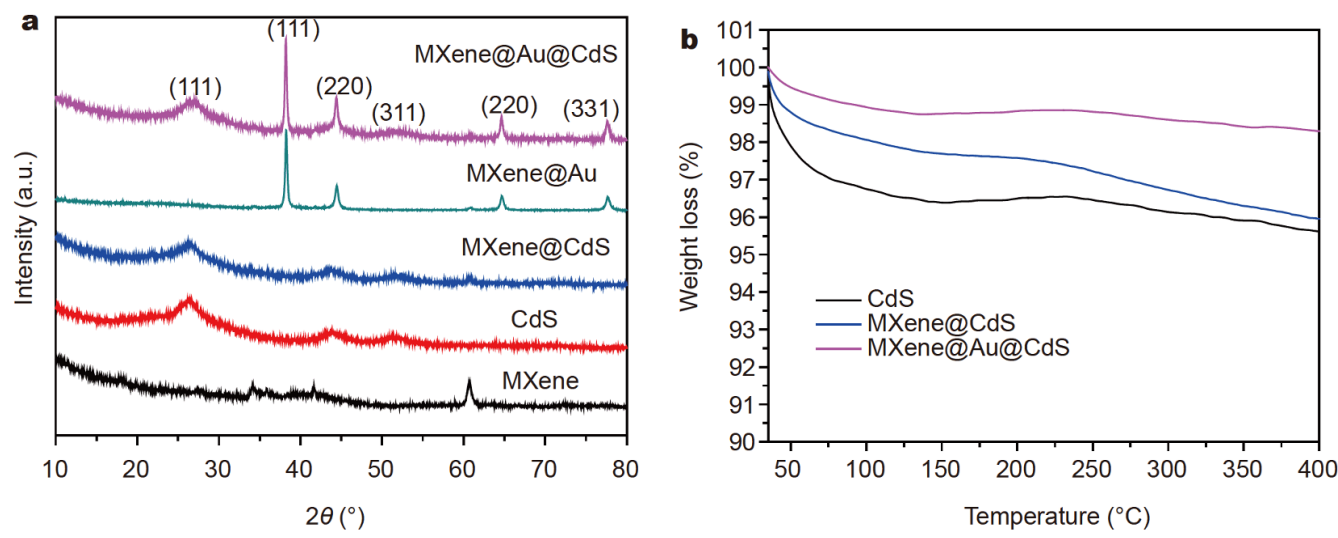

Figure 4 XRD patterns (a) and TG curves (b) of prepared MXene composites.
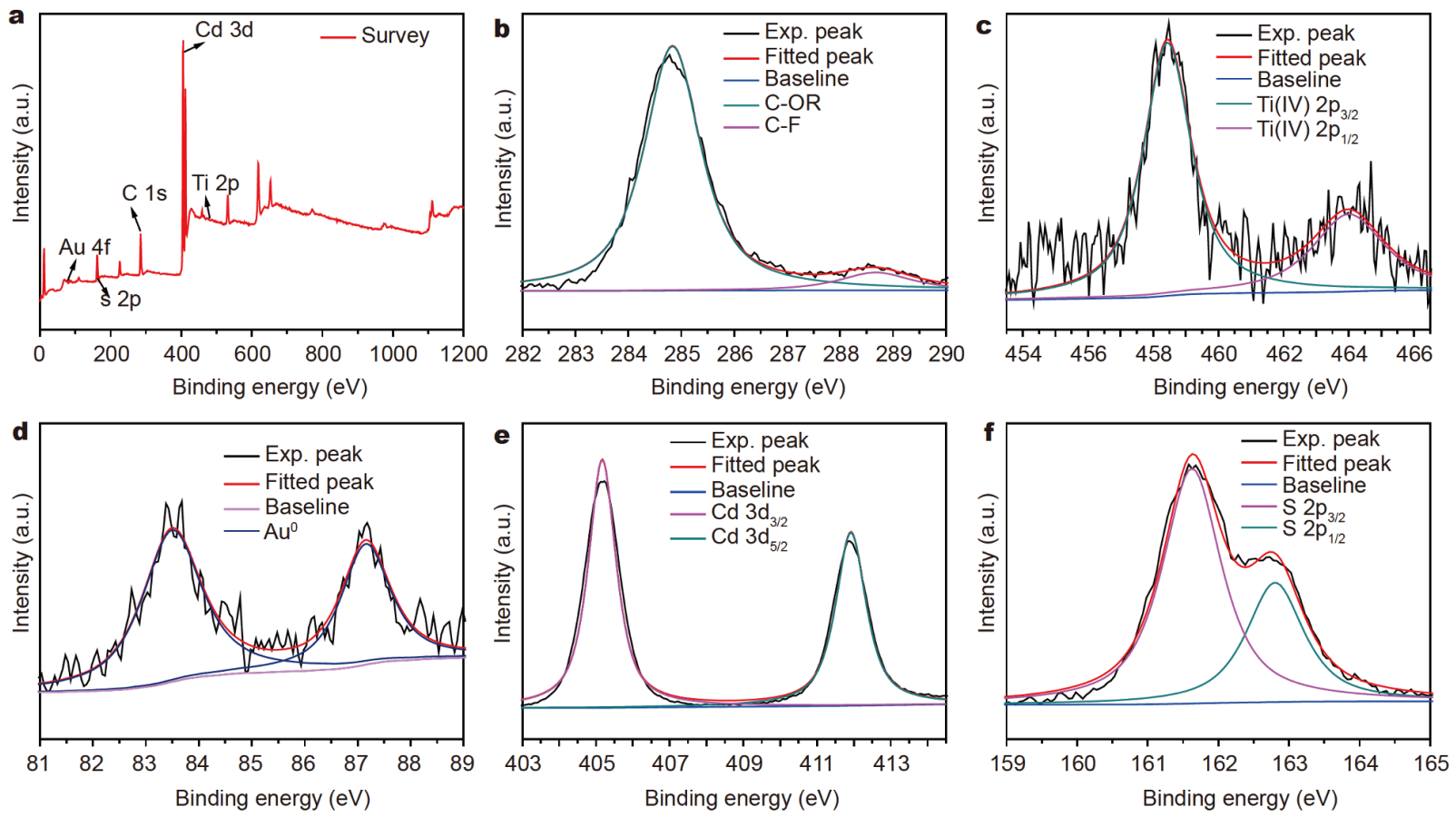

Figure 5 XPS profiles of the MXene@Au@CdS (a); C/Ti/Au/Cd/S bonding states in the MXene@Au@CdS (b-f).

hances the thermal stability of the material.

The chemical interface binding state of MXene $@$ Au@CdS was demonstrated by XPS, as shown in Fig. 5a-f. The $\mathrm{C} 1 \mathrm{~s}$ spectrum shows two distinct peaks at 284.84 and $288.67 \mathrm{eV}$, respectively, due to the $\mathrm{C}-\mathrm{OR}$ and $\mathrm{C}-\mathrm{F}$ bonds [37]. The peaks at 458.44 and $464.01 \mathrm{eV}$ on the Ti $2 \mathrm{p}$ spectrum are attributed to the $2 \mathrm{p}_{3 / 2}$ and $2 \mathrm{p}_{1 / 2}$ peaks of $\mathrm{Ti}_{3} \mathrm{C}_{2}$ [38]. For the $\mathrm{Au} 4 \mathrm{f}$ spectrum, two characteristic peaks of $\mathrm{Au} 0$ in the $\mathrm{Au} 4 \mathrm{f}_{7 / 2}$ and $\mathrm{Au} 4 \mathrm{f}_{5 / 2}$ states can be observed at 83.49 and $87.15 \mathrm{eV}$ [39]. In addition, the binding energies of $\mathrm{Cd} 3 \mathrm{~d}$ (Fig. 5e) and S 2p (Fig. 5f) are consistent with the data of CdS [40].

A nitrogen adsorption-desorption measurement was performed on the obtained samples to observe the specific surface area and porosity, as shown in Fig. 6. Pure MXene exhibits a typical $\mathrm{H} 3$ isotherm, indicating its mesoporous properties. Table 1 lists the Brunauer-Emmett-Teller (BET) surface areas, pore volumes and pore sizes of the prepared samples. The BET surface area, pore volume and pore size of MXene (MXene@Au@CdS) are $22.2672(34.5170) \mathrm{m}^{2} \mathrm{~g}^{-1}, 0.0563(0.0622) \mathrm{cm}^{3} \mathrm{~g}^{-1}$ and $9.7948(7.3110) \mathrm{nm}$, respectively, indicating that loading 

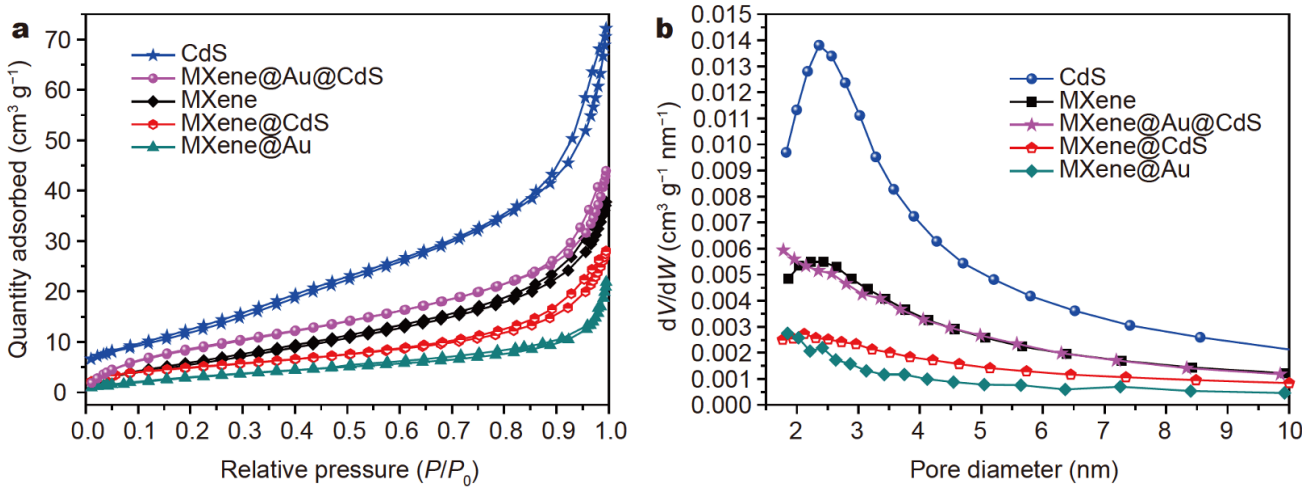

Figure $6 \mathrm{~N}_{2}$ adsorption-desorption curves (a) and the pore size distributions (b) of MXene, MXene@Au, MXene@CdS, MXene@Au@CdS and CdS.

Table 1 The BET data of as-prepared materials

\begin{tabular}{cccc}
\hline Catalysts & $\begin{array}{c}\text { Surface area } \\
\left(\mathrm{m}^{2} \mathrm{~g}^{-1}\right)\end{array}$ & $\begin{array}{c}\text { Pore volume } \\
\left(\mathrm{cm}^{3} \mathrm{~g}^{-1}\right)\end{array}$ & Pore size $(\mathrm{nm})$ \\
\hline MXene & 22.2672 & 0.0563 & 9.7948 \\
MXene@Au & 12.2502 & 0.0316 & 9.4052 \\
MXene@CdS & 17.8133 & 0.0421 & 9.0111 \\
MXene@Au@CdS & 34.5170 & 0.0622 & 7.3110 \\
CdS & 45.4100 & 0.1096 & 9.1249 \\
\hline
\end{tabular}

with Au@CdS nanoparticles, the MXene keeps a porous structure and possesses a relatively large specific surface area, which can provide some active sites for hydrogen production reactions.

As shown in Fig. 7, the MXene, MXene@Au, MXene @CdS, MXene@Au@CdS and CdS were investigated by UV-visible DRS spectra. As the wavelength increases, the absorption intensity of MXene gradually decreases, which can be mainly attributed to the unique absorption of carbonaceous materials [41]. In the DRS spectrum, the absorbance intensity of MXene is relatively low due to the small amount of MXene added. In addition, the CdS sample shows a steep absorption peak at $475 \mathrm{~nm}$ as expected, as shown in Fig. 7a. The absorption band of MXene@CdS composite is concentrated at about $475 \mathrm{~nm}$, showing the characteristic of CdS. The curve of the obtained MXene@Au@CdS shows spectral characteristics of $\mathrm{CdS}$ and MXene. Moreover, since the amount of MXene added in the process of self-reduction reaction is relatively excessive, there is a shielding effect on the absorption peak of the formed Au nanoparticles. Thus, there is no obvious surface plasmon resonance (SPR) peak for Au nanoparticles in the DRS results, as shown in Fig. 7b. The above results indicate that the prepared ternary composite MXene@Au@CdS effectively enhances the absorption of CdS in visible light range, beneficial for
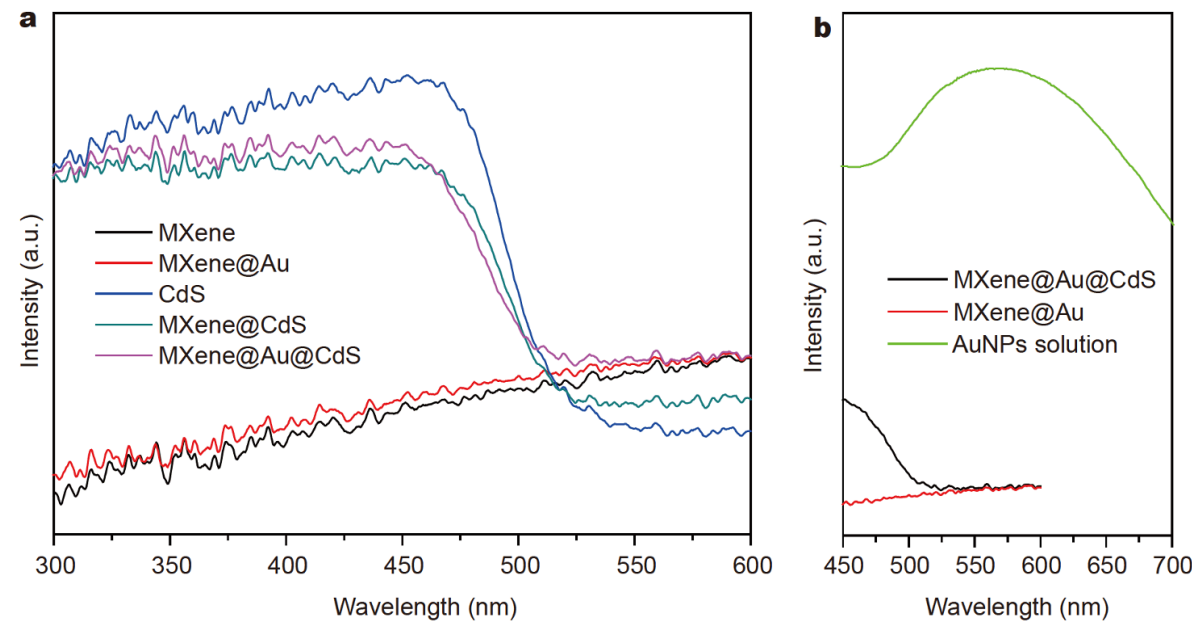

Figure 7 UV-vis DRS spectra of MXene, MXene@Au, MXene@CdS, and MXene@Au@CdS (a); enlarged curves of MXene@Au, MXene@Au@CdS and AuNPs solution (b). 
the subsequent photocatalytic hydrogen production reaction $[42,43]$.

\section{Photocatalytic performance of the MXene@Au@CdS}

According to literature report, under visible light irradiation $(\lambda>420 \mathrm{~nm})$, the activity of photocatalytic hydrogen production has been greatly improved [44-46]. Thus, the present prepared samples were evaluated for photocatalytic $\mathrm{H}_{2}$ release in aqueous $\mathrm{Na}_{2} \mathrm{~S}$ and $\mathrm{Na}_{2} \mathrm{SO}_{3}$ solutions under visible light irradiation, with the results of hydrogen evolution tests shown in Fig. 8a. The amount of released hydrogen was not detected in the absence of photocatalyst or light irradiation. In addition, the evolution of hydrogen from MXene@Au is inconspicuous, indicating that the nanocomposite MXene@Au has weak photocatalytic hydrogen evolution activity. Both of CdS composite materials show higher photocatalytic hydrogen production activity than pure CdS. The amount of hydrogen generated gradually increases from CdS, MXene @CdS, to MXene@Au@CdS ternary nanocomposite,. The composite MXene@CdS has a hydrogen production rate of $12,340.50 \mu \mathrm{mol} \mathrm{g}^{-1} \mathrm{~h}^{-1}$, while the MXene@Au@CdS shows a hydrogen production rate of $17,070.43 \mu \mathrm{mol} \mathrm{g}^{-1} \mathrm{~h}^{-1}$, which corresponds to 1.336 and 1.85 times that of CdS $\left(9234.46 \mu \mathrm{mol} \mathrm{g}^{-1} \mathrm{~h}^{-1}\right)$, respectively. This is because CdS forms a stronger bond with MXene and MXene@Au during hydrothermal reactions, which greatly promotes the rapid transfer of interface charge, highlighting the great potential of MXene. Moreover the powerful SPR with the sensitizer Au can greatly expand the optical response range of CdS. In addition, MXene is inactive against $\mathrm{H}_{2}$ production under visible light irradiation, further verifying its role as a cocatalyst rather than a photocatalyst. In order to investigate the stability of MXene@Au@CdS, cyclic hydrogen evolution experiments were performed, as shown in Fig. 8b. The MXene@Au@CdS shows relatively high stability and durability after four consecutive cycle tests.

In order to study the overall charge transfer efficiency and surface charge transfer efficiency of the obtained
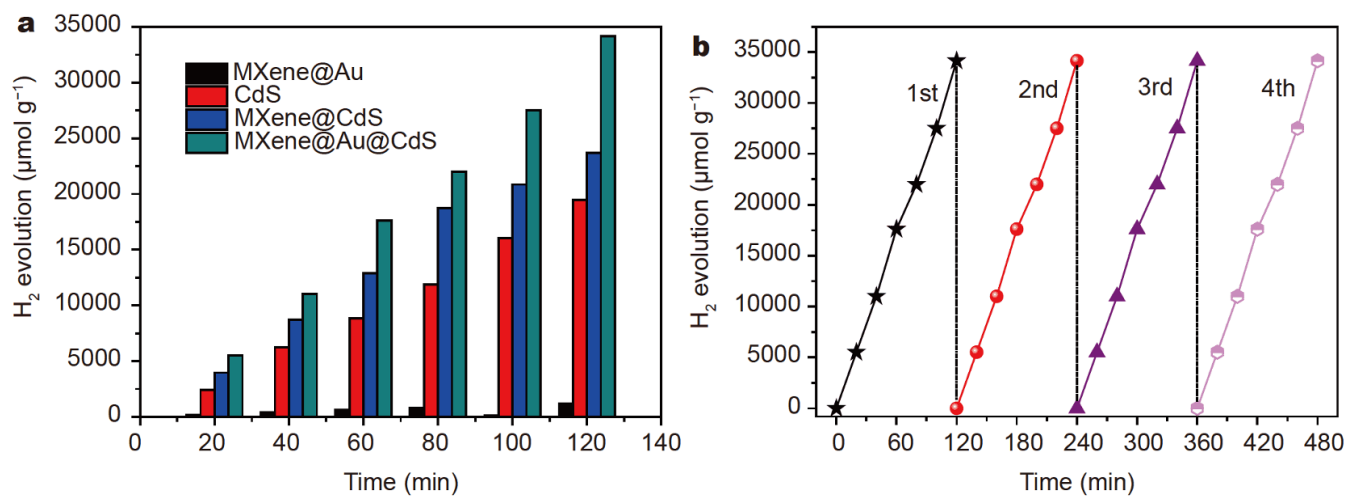

Figure 8 Hydrogen evolution profiles of CdS and MXene-based composites under visible-light illumination (a); $4 \mathrm{mg}$ of the photocatalyst was dispersed in a mixed aqueous solution of $80 \mathrm{~mL}$ of $0.35 \mathrm{~mol} \mathrm{~L}^{-1} \mathrm{Na}_{2} \mathrm{~S}$ and $0.25 \mathrm{~mol} \mathrm{~L}^{-1} \mathrm{Na}_{2} \mathrm{SO}_{3}$; cycle runs for the photocatalytic $\mathrm{H}_{2}$ production over MXene@Au@CdS nanocomposite (b).
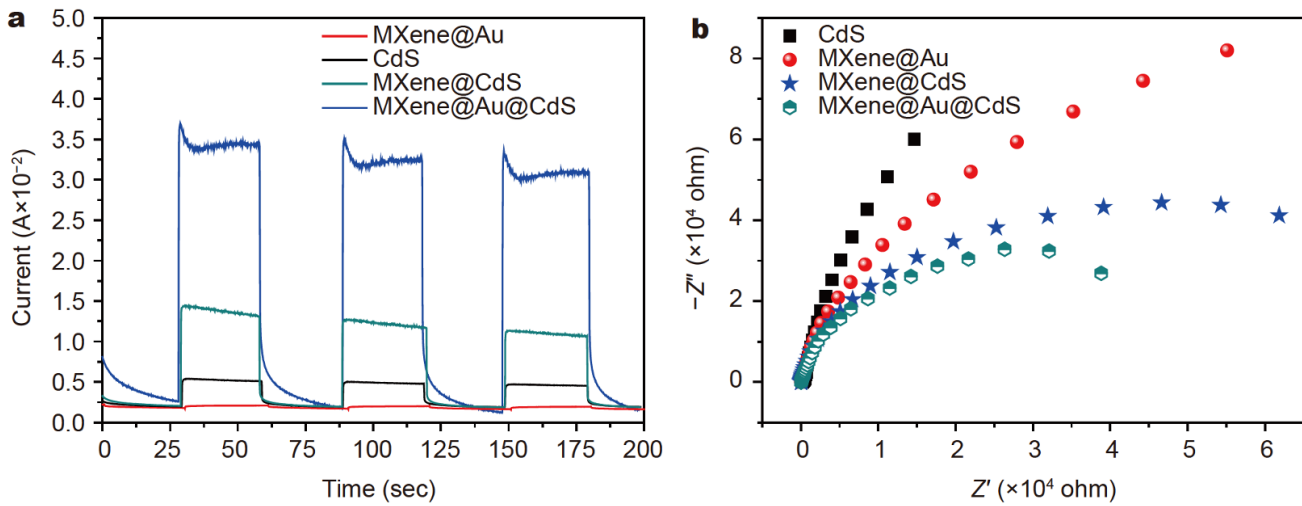

Figure 9 (a) i-t curves, (b) EIS of MXene, MXene@Au, MXene@CdS, MXene@Au@CdS and CdS. 


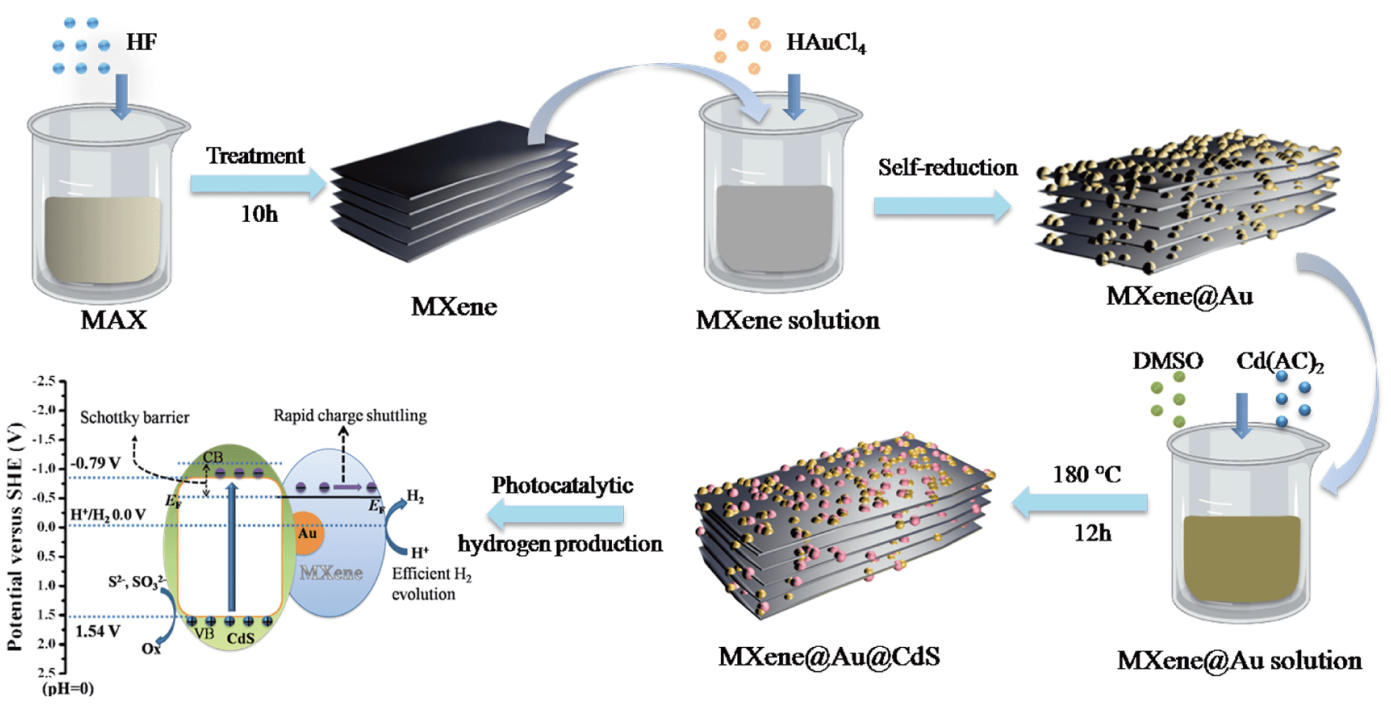

Figure 10 Schematic of the MXene@Au@CdS preparation flow chart and energy band structure diagram.

nanocomposites, the transient photocurrent (TPC) response and EIS tests were performed, as shown in Fig. 9. The anode photocurrent increases during illumination and disappears without illumination (Fig. 9a). The MXene@Au@CdS as the photoanode produces higher photocurrent than other samples, demonstrating that MXene@Au@CdS has higher charge separation efficiency. At the same time, the photocurrent is very stable in continuous on/off cycles. Under visible light irradiation, since the semicircular diameter of MXene@Au@CdS is smaller than those of other three materials, showing that the interface charge transfer resistance of MXene@Au@CdS is smaller, as shown in Fig. 9b. Therefore, it can be concluded that MXene@Au@CdS has better interface charge transfer capability on the surface than other samples.

The illustration of preparation and hydrogen production for the MXene@Au@CdS is shown in Fig. 10. First, a certain amount of concentrated HF solution was added to $\mathrm{Ti}_{3} \mathrm{AlC}_{2}$ suspension at room temperature, and $\mathrm{Al}$ layer in the $\mathrm{Ti}_{3} \mathrm{AlC}_{2}$ was etched to obtain $\mathrm{Ti}_{3} \mathrm{C}_{2}$ MXene. Next, a certain amount of $\mathrm{HAuCl}_{4}$ solution was added to the $\mathrm{Ti}_{3} \mathrm{C}_{2}$ MXene suspension under stirring to trigger the selfreduction. And then MXene@Au composite was obtained after a suitable reaction time. Subsequently MXene@Au powder was added to DMSO with stirring for about $20 \mathrm{~min}$. Then, appropriate amount of $\mathrm{Cd}\left(\mathrm{CH}_{3} \mathrm{CO}_{2}\right)_{2}$ solid was added to the above suspension, and continuously stirred for $2 \mathrm{~h}$. After that, the mixed solution was poured into an autoclave, which was heated up to $180^{\circ} \mathrm{C}$ and kept for 12 h. Finally, ternary MXene@Au@CdS was obtained.
A reasonable mechanism of electron-hole separation for the MXene@Au@CdS is proposed, as shown in Fig. 10. $\mathrm{CdS}$ is excited by visible light to generate electrons in the conduction band (CB) and holes in the valence band (VB) [46-48]. Since the original $E_{\mathrm{F}}$ of $\mathrm{n}$-type $\mathrm{CdS}$ is much more negative than the original $E_{\mathrm{F}}$ of MXene, the close contact between CdS and MXene will lead the electrons to transfer from CdS to MXene. At the same time, a fixed positive charge remains near the interface of CdS and MXene to form holes. Therefore, a Schottky junction is formed between MXene and CdS. Finally, the terminal photoelectrons of functional group $-\mathrm{O}$ in MXene can effectively reduce protons in aqueous solution by releasing $\mathrm{H}_{2}$ gas. The electrons transfer by two paths. One is that the Schottky junction effectively captures the lightsensing electrons, which exist as an electron trap. The other is accelerated by the strong SPR absorption of $\mathrm{Au}$ which could extend the response spectrum of CdS [49]. This study demonstrates a new clue for the design of catalytic materials [50-58] and self-assembled functionalized nanocomposites [59-65].

\section{CONCLUSIONS}

In summary, a new ternary nanocomposite MXene $@$ @u@CdS was designed and applied in the field of photocatalytic hydrogen production, showing high catalytic activity. Under visible light irradiation, the hydrogen production rate of the MXene@Au@CdS is $17,070.43 \mu \mathrm{mol} \mathrm{g}^{-1} \mathrm{~h}^{-1}$, which is 1.85 times that of CdS. This nanocomposite not only avoids the problem of lack of rich surface functions to establish a strong connection 
with photocatalyst, but also achieves rapid transfer of interface charges and long-term stability. The present study provides a strategy for opening up a new application field for MXene-based catalytic composite material. The as-prepared stable and cost-effective semiconductor photocatalyst is expected to effectively realize solar water decomposition.

\section{Received 31 January 2020; accepted 11 March 2020; published online 30 April 2020}

1 Chen X, Shen S, Guo L, et al. Semiconductor-based photocatalytic hydrogen generation. Chem Rev, 2010, 110: 6503-6570

2 Kudo A, Miseki Y. Heterogeneous photocatalyst materials for water splitting. Chem Soc Rev, 2009, 38: 253-278

3 Xiao F, Zhou W, Sun B, et al. Engineering oxygen vacancy on rutile $\mathrm{TiO}_{2}$ for efficient electron-hole separation and high solardriven photocatalytic hydrogen evolution. Sci China Mater, 2018, 61: 822-830

4 Qin Z, Wang M, Li R, et al. Novel $\mathrm{Cu}_{3} \mathrm{P} / \mathrm{g}-\mathrm{C}_{3} \mathrm{~N}_{4} \mathrm{p}$-n heterojunction photocatalysts for solar hydrogen generation. Sci China Mater, 2018, 61: 861-868

5 Tang Y, Zhou P, Chao Y, et al. Face-to-face engineering of ultrathin Pd nanosheets on amorphous carbon nitride for efficient photocatalytic hydrogen production. Sci China Mater, 2019, 62: 351-358

6 Zhang GQ, Ou W, Xu YS. Fluorescein supramolecular nanosheets: A novel organic photocatalyst for visible-light-driven $\mathrm{H}_{2}$ evolution from water. Sci China Mater, 2018, 61: 1001-1006

7 Zhang M, Luo Z, Zhou M, et al. Photocatalytic water oxidation by layered Co/h-BCN hybrids. Sci China Mater, 2015, 58: 867-876

8 Bi W, Li X, Zhang L, et al. Molecular co-catalyst accelerating hole transfer for enhanced photocatalytic $\mathrm{H}_{2}$ evolution. Nat Commun, 2015, 6: 8647

9 Yin Y, Jin Z, Hou F. Enhanced solar water-splitting efficiency using core/sheath heterostructure $\mathrm{CdS} / \mathrm{TiO}_{2}$ nanotube arrays. Nanotechnology, 2007, 18: 495608

10 Wang Y, Wang F, He J. Controlled fabrication and photocatalytic properties of a three-dimensional $\mathrm{ZnO}$ nanowire/reduced graphene oxide/CdS heterostructure on carbon cloth. Nanoscale, 2013, 5: 11291-11297

11 He K, Li M, Guo L. Preparation and photocatalytic activity of PANI-CdS composites for hydrogen evolution. Int J Hydrogen Energy, 2012, 37: 755-759

12 Chang $\mathrm{Y}, \mathrm{Yu} \mathrm{K}$, Zhang $\mathrm{C}$, et al. Ternary CdS/Au/3DOM-SrTiO composites with synergistic enhancement for hydrogen production from visible-light photocatalytic water splitting. Appl Catal B-Environ, 2017, 215: 74-84

13 Tada H, Mitsui T, Kiyonaga T, et al. All-solid-state Z-scheme in $\mathrm{CdS}-\mathrm{Au}-\mathrm{TiO}_{2}$ three-component nanojunction system. Nat Mater, 2006, 5: 782-786

14 Zeng Z, Li T, Li YB, et al. Plasmon-induced photoelectrochemical water oxidation enabled by in situ layer-by-layer construction of cascade charge transfer channel in multilayered photoanode. J Mater Chem A, 2018, 6: 24686-24692

15 Zeng $\mathrm{Z}$, Li YB, Chen $\mathrm{S}$, et al. Insight into the charge transport correlation in $\mathrm{Au}_{x}$ clusters and graphene quantum dots deposited on $\mathrm{TiO}_{2}$ nanotubes for photoelectrochemical oxygen evolution. J Mater Chem A, 2018, 6: 11154-11162
16 Li T, Huang MH, Li YB, et al. General self-assembly of metal/metal chalcogenide heterostructures initiated by a surface linker: modulating tunable charge flow toward versatile photoredox catalysis. J Mater Chem A, 2019, 7: 21182-21194

17 Li T, Li YB, Dai XC, et al. Ligand-triggered tunable charge transfer toward multifarious photoreduction catalysis. J Phys Chem C, 2019, 123: 4701-4714

18 Naguib M, Mashtalir O, Carle J, et al. Two-dimensional transition metal carbides. ACS Nano, 2012, 6: 1322-1331

19 Ghidiu M, Lukatskaya MR, Zhao MQ, et al. Conductive twodimensional titanium carbide 'clay' with high volumetric capacitance. Nature, 2014, 516: 78-81

20 Anasori B, Lukatskaya MR, Gogotsi Y. 2D metal carbides and nitrides (MXenes) for energy storage. Nat Rev Mater, 2017, 2: 1-7

21 Yang H, Wang C, Hu F, et al. Atomic-scale Pt clusters decorated on porous $\alpha-\mathrm{Ni}(\mathrm{OH})_{2}$ nanowires as highly efficient electrocatalyst for hydrogen evolution reaction. Sci China Mater, 2017, 60: 11211128

22 Ran J, Gao G, Li FT, et al. $\mathrm{Ti}_{3} \mathrm{C}_{2}$ MXene co-catalyst on metal sulfide photo-absorbers for enhanced visible-light photocatalytic hydrogen production. Nat Commun, 2017, 8: 13907

23 Férey G, Mellot-Draznieks C, Serre C, et al. A chromium terephthalate-based solid with unusually large pore volumes and surface area. Science, 2005, 309: 2040-2042

24 Lukatskaya MR, Mashtalir O, Ren CE, et al. Cation intercalation and high volumetric capacitance of two-dimensional titanium carbide. Science, 2013, 341: 1502-1505

25 Li K, Jiao T, Xing R, et al. Fabrication of tunable hierarchical MXene@AuNPs nanocomposites constructed by self-reduction reactions with enhanced catalytic performances. Sci China Mater, 2018, 61: 728-736

26 Xiang Q, Yu J, Jaroniec M. Synergetic effect of $\mathrm{MoS}_{2}$ and graphene as cocatalysts for enhanced photocatalytic $\mathrm{H}_{2}$ production activity of $\mathrm{TiO}_{2}$ nanoparticles. J Am Chem Soc, 2012, 134: 6575-6578

27 Huang X, Wang R, Jiao T, et al. Facile preparation of hierarchical AgNP-loaded MXene $/ \mathrm{Fe}_{3} \mathrm{O}_{4}$ /polymer nanocomposites by electrospinning with enhanced catalytic performance for wastewater treatment. ACS Omega, 2019, 4: 1897-1906

28 Zhang J, Xi J, Ji Z. Mo $+\mathrm{N}$ codoped $\mathrm{TiO}_{2}$ sheets with dominant $\{001\}$ facets for enhancing visible-light photocatalytic activity. J Mater Chem, 2012, 22: 17700-17708

29 Huo S, Duan P, Jiao T, et al. Self-assembled luminescent quantum dots to generate full-color and white circularly polarized light. Angew Chem Int Ed, 2017, 56: 12174-12178

30 Zhao X, Ma K, Jiao T, et al. Fabrication of hierarchical layer-bylayer assembled diamond-based core-shell nanocomposites as highly efficient dye absorbents for wastewater treatment. Sci Rep, 2017, 7: 44076

31 Wang C, Sun S, Zhang L, et al. Facile preparation and catalytic performance characterization of AuNPs-loaded hierarchical electrospun composite fibers by solvent vapor annealing treatment. Colloids Surfs A-Physicochem Eng Aspects, 2019, 561: 283-291

32 Feng $\mathrm{Y}$, Jiao T, Yin J, et al. Facile preparation of carbon nanotube$\mathrm{Cu}_{2} \mathrm{O}$ nanocomposites as new catalyst materials for reduction of $p$ nitrophenol. Nanoscale Res Lett, 2019, 14: 78

33 Guo R, Jiao T, Li R, et al. Sandwiched $\mathrm{Fe}_{3} \mathrm{O}_{4} /$ carboxylate graphene oxide nanostructures constructed by layer-by-layer assembly for highly efficient and magnetically recyclable dye removal. ACS Sustain Chem Eng, 2018, 6: 1279-1288

34 Ji Y, Ghosh K, Shu XZ, et al. Electrospun three-dimensional 
hyaluronic acid nanofibrous scaffolds. Biomaterials, 2006, 27: 3782-3792

35 Liu K, Xing R, Li Y, et al. Mimicking primitive photobacteria: sustainable hydrogen evolution based on peptide-porphyrin coassemblies with a self-mineralized reaction center. Angew Chem Int Ed, 2016, 55: 12503-12507

36 Liu K, Yuan C, Zou Q, et al. Self-assembled zinc/cystine-based chloroplast mimics capable of photoenzymatic reactions for sustainable fuel synthesis. Angew Chem Int Ed, 2017, 56: 7876-7880 Rakhi RB, Ahmed B, Hedhili MN, et al. Effect of postetch annealing gas composition on the structural and electrochemical properties of $\mathrm{Ti}_{2} \mathrm{CT}_{x}$ MXene electrodes for supercapacitor applications. Chem Mater, 2015, 27: 5314-5323

38 Dang BHQ, Rahman M, MacElroy D, et al. Evaluation of microwave plasma oxidation treatments for the fabrication of photoactive un-doped and carbon-doped $\mathrm{TiO}_{2}$ coatings. Surf Coatings Tech, 2012, 206: 4113-4118

39 Chen L, He BY, He S, et al. Fe-Ti oxide nano-adsorbent synthesized by co-precipitation for fluoride removal from drinking water and its adsorption mechanism. Powder Tech, 2012, 227: 3-8

40 Li B, Zhao Z, Gao F, et al. Mesoporous microspheres composed of carbon-coated $\mathrm{TiO}_{2}$ nanocrystals with exposed $\{001\}$ facets for improved visible light photocatalytic activity. Appl Catal B-Environ, 2014, 147: 958-964

41 Zhang L, Pan X, Xia L, et al. $\mathrm{TiO}_{2}-\mathrm{Ti}_{3} \mathrm{C}_{2}$ composites with Pt decoration as efficient photocatalysts for ethylene oxidation under near infrared light irradiation. Chin J Struc Chem, 2018, 37: 14571469

42 Tang Q, Zhou Z, Shen P. Are MXenes promising anode materials for li ion batteries? Computational studies on electronic properties and li storage capability of $\mathrm{Ti}_{3} \mathrm{C}_{2}$ and $\mathrm{Ti}_{3} \mathrm{C}_{2} \mathrm{X}_{2}(\mathrm{X}=\mathrm{F}, \mathrm{OH})$ monolayer. J Am Chem Soc, 2012, 134: 16909-16916

43 Sun $\mathrm{D}$, Wang $\mathrm{M}, \mathrm{Li} \mathrm{Z}$, et al. Two-dimensional $\mathrm{Ti}_{3} \mathrm{C}_{2}$ as anode material for Li-ion batteries. Electrochem Commun, 2014, 47: 8083

44 Xu J, Yang WM, Huang SJ, et al. CdS core-Au plasmonic satellites nanostructure enhanced photocatalytic hydrogen evolution reaction. Nano Energy, 2018, 49: 363-371

45 Song J, Xing R, Jiao T, et al. Crystalline dipeptide nanobelts based on solid-solid phase transformation self-assembly and their polarization imaging of cells. ACS Appl Mater Interfaces, 2018, 10: 2368-2376

46 Xing R, Jiao T, Yan L, et al. Colloidal gold-collagen protein coreshell nanoconjugate: one-step biomimetic synthesis, layer-by-layer assembled film, and controlled cell growth. ACS Appl Mater Interfaces, 2015, 7: 24733-24740

47 Wen M, Mori K, Kamegawa T, et al. Amine-functionalized MIL$101(\mathrm{Cr})$ with imbedded platinum nanoparticles as a durable photocatalyst for hydrogen production from water. Chem Commun, 2014, 50: 11645-11648

48 He J, Yan Z, Wang J, et al. Significantly enhanced photocatalytic hydrogen evolution under visible light over CdS embedded on metal-organic frameworks. Chem Commun, 2013, 49: 6761-6763

49 Ding X, Li Y, Zhao J, et al. Enhanced photocatalytic $\mathrm{H}_{2}$ evolution over $\mathrm{CdS} / \mathrm{Au} / \mathrm{g}-\mathrm{C}_{3} \mathrm{~N}_{4}$ composite photocatalyst under visible-light irradiation. APL Mater, 2015, 3: 104410

50 Cai C, Wang R, Liu S, et al. Synthesis of self-assembled phytic acidMXene nanocomposites via a facile hydrothermal approach with elevated dye adsorption capacities. Colloids Surfs A-Physicochem Eng Aspects, 2020, 589: 124468
51 Yin J, Zhan F, Jiao T, et al. Highly efficient catalytic performances of nitro compounds via hierarchical PdNPs-loaded MXene/polymer nanocomposites synthesized through electrospinning strategy for wastewater treatment. Chin Chem Lett, 2020, 31: 992-995

52 Feng Y, Yin J, Liu S, et al. Facile synthesis of $\mathrm{Ag} / \mathrm{Pd}$ nanoparticleloaded poly(ethylene imine) composite hydrogels with highly efficient catalytic reduction of 4-nitrophenol. ACS Omega, 2020, 5: 3725-3733

53 Zhao J, Yin J, Zhong J, et al. Facile preparation of a self-assembled Artemia cyst shell- $-\mathrm{TiO}_{2}-\mathrm{MoS}_{2}$ porous composite structure with highly efficient catalytic reduction of nitro compounds for wastewater treatment. Nanotechnology, 2020, 31: 085603

54 Zhu J, Wang R, Geng R, et al. A facile preparation method for new two-component supramolecular hydrogels and their performances in adsorption, catalysis, and stimuli-response. RSC Adv, 2019, 9: 22551-22558

55 Wang C, Yin J, Han S, et al. Preparation of palladium nanoparticles decorated polyethyleneimine/polycaprolactone composite fibers constructed by electrospinning with highly efficient and recyclable catalytic performances. Catalysts, 2019, 9: 559

$56 \mathrm{Xu} \mathrm{Y}$, Wang R, Zheng $\mathrm{Y}$, et al. Facile preparation of self-assembled $\mathrm{Ni} / \mathrm{Co}$ phosphates composite spheres with highly efficient HER electrocatalytic performances. Appl Surf Sci, 2020, 509: 145383

57 Geng $\mathrm{R}$, Yin J, Zhou J, et al. In situ construction of $\mathrm{Ag} / \mathrm{TiO}_{2} / \mathrm{g}$ $\mathrm{C}_{3} \mathrm{~N}_{4}$ heterojunction nanocomposite based on hierarchical coassembly with sustainable hydrogen evolution. Nanomaterials, 2020, 10: 1

58 Zhan F, Yin J, Zhou J, et al. Facile preparation and highly efficient catalytic performances of Pd-Cu bimetallic catalyst synthesized via seed-mediated method. Nanomaterials, 2020, 10: 6

59 Wang R, Yan X, Ge B, et al. Facile preparation of self-assembled black phosphorus-dye composite films for chemical gas sensors and surface-enhanced Raman scattering performances. ACS Sustain Chem Eng, 2020, 8: 4521-4536

60 Ma K, Wang R, Rao Y, et al. Langmuir-Blodgett films of two chiral perylene bisimide-based molecules: aggregation and supramolecular chirality. Colloids Surfs A-Physicochem Eng Aspects, 2020, 591: 124563

61 He Y, Wang R, Sun C, et al. Facile synthesis of self-assembled NiFe layered double hydroxide-based azobenzene composite films with photoisomerization and chemical gas sensor performances. ACS Omega, 2020, 5: 3689-3698

62 Song J, Yuan C, Jiao T, et al. Multifunctional antimicrobial biometallohydrogels based on amino acid coordinated self-assembly. Small, 2020, 16: 1907309

63 Li H, Yin J, Meng Y, et al. Nickel/cobalt-containing polypyrrole hydrogel-derived approach for efficient ORR electrocatalyst. Colloids Surfs A-Physicochem Eng Aspects, 2020, 586: 124221

64 Meng Y, Yin J, Jiao T, et al. Self-assembled copper/cobaltcontaining polypyrrole hydrogels for highly efficient ORR electrocatalysts. J Mol Liquids, 2020, 298: 112010

65 He Y, Wang R, Jiao T, et al. Facile preparation of self-assembled layered double hydroxide-based composite dye films as new chemical gas sensors. ACS Sustain Chem Eng, 2019, 7: 10888-10899

Acknowledgements This work was supported by the National Natural Science Foundation of China (21872119), the Talent Engineering Training Funding Project of Hebei Province (A201905004), and the Research Program of the College Science and Technology of Hebei Province (ZD2018091). 
Author contributions Yin J, Jiao T, and Jiang G designed the project and performed the experiments. Zhan F, Wang W, Zhang G, Jiao J, Zhang Q, Gu J and Peng Q characterized the materials and discussed the results of the experiments. All the authors contributed to the general discussion.

Conflict of interest The authors declare no conflict of interest.

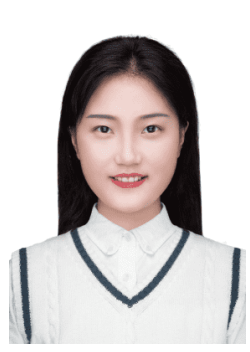

Juanjuan Yin is a $\mathrm{PhD}$ candidate supervised by Prof. Tifeng Jiao at the School of Environmental and Chemical Engineering of Yanshan University. Her current research interest focuses on MXene-based nanocomposites for photo- and electrocatalytic hydrogen production.

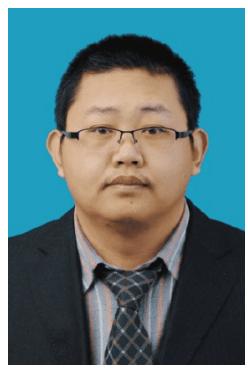

Tifeng Jiao received his $\mathrm{PhD}$ in physical chemistry from the Institute of Chemistry, Chinese Academy of Sciences (CAS). He was a postdoctoral fellow of CNRS (Centre National de la Recherche Scientifique) with A.P. Girard-Egrot (Université Claude Bernard Lyon 1, France). Currently, he is a full professor and vice director of the School of Environmental and Chemical Engineering, Yanshan University. His current research interests include the synthesis of new self-assembled nanostructured materials and nanocomposites, and their related properties.

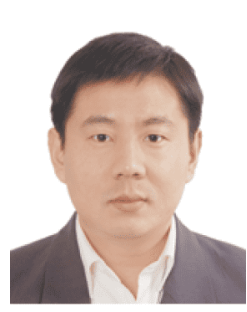

Guiyuan Jiang received his BE and Master degrees from China University of Petroleum, Beijing and $\mathrm{PhD}$ degree from the Institute of Chemistry, CAS in 2000, 2003 and 2006, respectively. He joined China University of Petroleum, Beijing in 2006, and became a full professor in 2012. He was a visiting postdoctor at the University of California, Riverside in 2010, and visiting scholar in Tsinghua University in 2013-2014. His research interest mainly focuses on the energy catalysis, including catalytic conversion of light hydrocarbons and artificial photosynthesis.

\section{自组装纳米复合材料 MXene@Au@CdS 的制备及 其光催化制氢活性}

\author{
尹娟娟 ${ }^{1,2}$, 展方可 ${ }^{2}$, 焦体峰 ${ }^{1,2 *}$, 王文涵 ${ }^{3}$, 张广聪, 焦菁华 ${ }^{2}$, \\ 姜桂元 ${ }^{*^{*}}$, 张庆瑞 ${ }^{2}$, 谷建民 ${ }^{2}$, 彭秋明 ${ }^{1}$
}

摘要 光催化制氢被认为是一种有效获得清洁可持续能源的方法. 常规光催化制氢使用的助催化剂 (如 $\mathrm{Pt}$ ) 具有成本高和难以获得的 缺点. 本文设计并制备了三元纳米复合材料MXene@Au@CdS, 可用 于高效光催化制氢. MXene@ Au@CdS 的氢气产生率为 17070.43 $\mu \mathrm{mol} \mathrm{g} \mathrm{g}^{-1} \mathrm{~h}^{-1}$ (测试时间 $2 \mathrm{~h}$ ), 是纯CdS纳米材料的 1.85 倍. MXene $@ \mathrm{Au} @ \mathrm{CdS}$ 优异的制氢性能归因于: (i) MXene为 $\mathrm{Au}$ 和CdS纳米颗粒 提供了更多的活性吸附位点和反应中心. (ii) 金的强表面等离子体 共振协同效应使得 $\mathrm{CdS}$ 的光学响应范围增大. 本工作解决了光催化 剂表面官能团之间的固态连接问题, 并在制氢过程中实现了快速 界面电荷转移和长期稳定性. 